\title{
The recognition of faces, expressions, and moods
}

\author{
DEBRA COHEN-PAGER and LEONARD BROSGOLE \\ St. John's University, Jamaica, New York
}

\begin{abstract}
This was a recognition study, in which only one inspection face was followed by a recognition test 5 days later. The level of facial, expressional, and mood recognition was assessed over six different conditions. Face recognition was better than expressional and mood recognition when familiar faces were used in the test phase of the study. However, the memory of the affective expression of a previously seen face was comparable to facial memory when prompted by the use of unfamiliar faces in the recognition test.
\end{abstract}

Galper and Hochberg (1971) used a standard face recognition design, consisting of an inspection phase followed by a recognition test. Their test for recognition assessed both facial and expressional recognition over a series of trials. They found that face recognition was significantly better than expressional recognition. They suggested that the more invariant features of the face (e.g., eyebrows, hair style) are registered in memory more strongly than changing features that are used for conveying facial expression (e.g., the mouth). According to this line of reasoning, the recognition of facial identity should be vastly superior to the recognition of facial expression. The findings of Galper and Hochberg have been confirmed by Cohen-Pager and Brosgole (1992), who also propose a piecemeal interpretation of face perception.

However, there may be another way to comprehend face perception. Obviously, certain feature cues are used for purposes of identification. However, expressional ones are also used for assessing mood. It is entirely possible that all of the cues transmitted by a face are incorporated into memory, but that the cues represented in consciousness consist only of the features that pertain to the requirements of the task at hand. That is to say, there may be a shift in memory with changes in the set of the observer. Feature cues may emerge with the need to identify a face, and expressional cues may emerge with the need to assess mood.

There is a serious problem involved in the attempt to determine a subject's level of expressional recognition. Through a life's history of having to identify people, every subject enters into a recognition task with a preexperimental set for identifying the face seen previously. As a result, the perceptual situation may be captured by the emergence of features that lead most readily to accurate facial identification. The dilemma is how to design a study to overcome the habituated use of feature cues, so that expressional memory can be accurately evaluated. Obvi-

This research is based on a dissertation that was submitted in partial fulfillment of the doctoral requirements at St. John's University. Requests for reprints should be sent to L. Brosgole, Psychology Laboratory, St. John's University, Jamaica, NY 11439. ously, the problem could be resolved by instructing subjects to try to remember the moods suggested by the pictures to be shown, but that would be absurd. Therefore, the following study was aimed at evaluating expressional memory by defeating the use of feature cues nonverbally.

Galper and Hochberg (1971) used a standard recognition design, which consisted of an inspection phase followed by a recognition test. Their test for recognition assessed both facial and expressional recognition over a series of trials. The level of face recognition was assessed by having the subjects distinguish between familiar and unfamiliar faces. The level of expressional recognition was determined by having the subjects distinguish between old and new expressions on a familiar face. In the present study, we employed essentially the same design, except that only one face was presented for inspection. This procedure eliminated the potential for intraserial interference among the inspection faces and reduced the memory load placed upon the subjects. Subsequent recognition tests were run over six different conditions, to assess the level of facial and expressional recognition after the exposure of a single picture. Different subjects were run in each condition. It was reasoned that if there is an essential difference between facial and expressional memory, the findings produced by the use of a series of inspection faces should be obtained with the exposure of a single picture.

\section{METHOD}

\section{Subjects}

Forty-four male and 76 female undergraduates, who ranged in age from 18 to 46 and had a mean age of 20 , were tested. The subjects were naive with respect to the purpose of the study. Each subject was assigned randomly to one of six groups, resulting in 20 subjects per group. All participants gave informed consent by signing an agreement and were treated in accordance with the ethical principles of the American Psychological Association.

\footnotetext{
Materials

The stimuli consisted of photographs used in a prior study by CohenPager and Brosgole (1992).

\section{Procedure}

All of the subjects viewed a photograph of either one neutral or one smiling target face in the inspection phase. The photograph was exposed
} 
for $7 \mathrm{sec}$, and the subjects were told to "look at the picture with the aim of later being able to remember it." Five days later, the subjects were tested with one pair, and the instructions were varied according to the specifics of the condition being run. Also, the particulars of the test pair varied by condition. Inspection and test pictures were varied over subjects and conditions. Each condition will be discussed separately.

The six conditions differed in terms of the recognition test only. In Condition 1, the subjects viewed the original target face along with a new face, both of which had the same expression as that of the target face when it was originally shown. The subjects were told to "point to the picture that you saw the other day." This condition was designed to test for face recognition.

In Condition 2, the subjects viewed two photographs of the original target face. In one, the face was smiling; the other had a neutral expression. Again, the subjects were asked to "point to the picture that you saw the other day." This condition was designed to test for expressional recognition in the absence of a prior inspection series.

The subjects in Condition 3 viewed the original target face with the opposite expression and a new face with the same expression as the original target face. The subjects were instructed to "point to the picture that you saw the other day." This condition was aimed at creating a conflict between facial and expressional recognition, in order to determine which took priority cognitively.

Conditions 4 and 5 were designed to assess the level of expressional recognition when the subjects were given an instructional set at the time of the recognition test to remember expression. Except for the instructions, both of these conditions were exactly the same as Condition 3 (the conflict condition).

In Condition 4, the test stimuli were placed on a table before each subject, and the subject was told to "point to the picture that feels the same, or has the same mood as the one you saw the other day." To control for the possibility that the subjects might have adopted a strategy to identify the stimulus seen before the receipt of the instructions, Condition 5 was run. This was the same as Condition 4, except that the instructions were given prior to the display of the test stimuli.

In Condition 6, the subjects viewed two new faces, each different from each other and of the sex opposite that of the target face originally viewed. One face was smiling; the other was neutral. The subjects were told to "point to the picture that feels the same, or has the same mood as the one you saw the other day." This condition was used to evaluate the use of expressional information in the complete absence of cues to facial identity.

\section{RESULTS}

There were six conditions, with 20 different subjects in each condition. The total number of correct responses was recorded for each condition. As previously noted, Condition 1 was directed at assessing the level of face recognition. In this condition, all of the subjects pointed to the face that they had seen previously $(p<.01$, according to the binomial test). The second condition was aimed at determining the level of expressional recognition, and 17 of the 20 subjects identified the expression that they had seen before $(p<.01)$. In Condition 3, a conflict was created between facial and expressional recognition. In this instance, 15 of the 20 subjects responded to the prior face, and 5 pointed to the familiar expression $(p<.05)$.

In Condition 4, only 10 of the 20 subjects responded to expressional cues when told to select the picture that felt the same, or had the same mood as the one seen previously. Even when these instructions were given prior to presentation of the test stimuli, as in Condition 5, only 11 of the 20 subjects responded appropriately. The responses were at chance level in both of these conditions.
Given these findings, it is interesting that 19 of the 20 subjects in Condition 6 responded to the familiar expression with the omission of cues to facial identity $(p<.01)$.

\section{DISCUSSION}

In a simplified study, Conditions 1 and 2 verified the results of Galper and Hochberg (1971), as well as those obtained previously by CohenPager and Brosgole (1992). In Condition 1, all of the subjects responded correctly, demonstrating perfect facial recognition. Although a significant number of subjects selected the previously seen expressions in Condition 2, some errors were committed. The two conditions combined indicated that expressional recognition was above chance, but that it was somewhat inferior to facial recognition. Condition 3 confirmed the results of the previous two treatments, by showing that a significant number of subjects selected facial over expressional cues in a free-choice situation.

The results of Conditions 4 and 5 were very surprising, particularly when compared with the data obtained from Condition 2 . Condition 2 revealed a high level of expressional recognition: $85 \%$ of the subjects responded correctly to expression. Yet the subjects in Conditions 4 and 5 performed at chance when having to select the mood seen previously. It is as if expression had registered as a feature in Condition 2, with no affective significance. That is, expression may have served as an ancillary cue for facial identification in Condition 2, communicating nothing about mood. Perhaps the very presentation of a familiar face captured the perceptual situation, generating an immediate set to identify that face by detecting features and making a template match of the present with the past. We evaluated this reasoning by running the sixth condition.

It may be recalled that in Condition 6, each inspection face was followed by two test faces of the opposite gender. One of the test faces had a familiar expression, and the other had an unfamiliar expression. The subjects were requested to point to the picture that "felt" the same as the one seen previously. This procedure defeated the emergence of a strategy to detect features, because the test faces were different from the prior inspection face. Nineteen of 20 subjects responded correctly to mood in this test situation. In addition, 18 of those 19 subjects voluntarily justified their choices by describing the expressional similarity between the inspection and the chosen test faces. Twelve of the subjects used words referring to mood (e.g., happy and glad vs. unhappy or no feeling), and the remaining 6 subjects used expressional words (smiling and laughing vs. neutral and no smile). Both the pointing data and the verbal reports obtained in Condition 6 demonstrated that mood registered when the target pictures were being inspected. Thus, the inferiority of expressional to facial recognition seems to be due to a retrieval problem created at the point of testing for recognition, rather than a failure for expression to be registered in memory at the time of inspection.

In summary, there were two main findings in this study. The first finding, evidenced in the data from Conditions 2, 4, and 5, indicated that facial expression can be registered independently of mood. The second finding, demonstrated in Condition 6, showed that mood can be registered independently of the specific features used to create a particular facial expression. These findings are supported by experiments performed with certain clinical populations. Kurucz, Feldmar, and Werner (1979) tested senile geriatrics and found that they were deficient in the recognition of facial affect even though they were sufficiently able to recognize familiar faces. In a follow-up study, Brosgole, Kurucz, PlaHovinsak, and Gumiela (1981) also found that senile elderly people were deficient in recognizing certain facial affective expressions, despite the fact that they were quite capable of discriminating between unfamiliar faces. In the same fashion, Gioia and Brosgole (1988) demonstrated that mentally handicapped subjects were better at recognizing facial expressions than facial affects, and PlaHovinsak (1989) found just the opposite with paranoid and chronic undifferentiated schizophrenics. Finally, Tranel, Damasio, and Damasio (1988) tested a small group of prosopagnosics and found that they were quite able to recognize facial expressions, age, and gender, even though they were unable to recognize familiar faces. All of these studies run on clinical populations seem to indicate that expression, mood, and identity are registered separately in memory and can be independent of one another. 
In conclusion, information regarding facial identity, expression, and mood seems to be registered with the very first exposure of an unfamiliar face. The superiority of facial to expressional recognition emerges at the time of testing, apparently due to a preexperimental set for subjects to identify faces rather than expressions. The recognition of mood is equivalent to the recognition of facial identity, once such a set is defeated.

\section{REFERENCES}

Brosgole, L., Kurucz, J., PlaHovinsak, T., \& Gumiela, E. (1981) On the mechanism underlying facial-affective agnosia in senile demented patients. International Journal of Neuroscience, 15, 207-215.

Cohen-Pager, D., \& Brosgole, L. (1992). The recognition of faces and expressions. Bulletin of the Psychonomic Society, 30, 191-193.

GALPER, R. E., \& HoCHBERG, J. (1971). Recognition memory for photographs of faces. American Journal of Psychology, 84, 351-354.
GioiA, J. V., \& Brosgole, L. (1988). Visual and auditory affect recognition in singly diagnosed mentally retarded patients, mentally retarded patients with autism, and normal young children. International Journal of Neuroscience, 43, 149-163.

Kurucz, J., Feldmar, G., \& Werner, W. (1979). Prosopo-affective agnosia as a symptom of cerebral organic disease. Journal of the American Geriatric Society, 27, 91-95.

PlaHovinsak, T. (1989). Facial, postural and auditory affect recognition in acute and remitted paranoid and nonparanoid schizophrenics. Unpublished doctoral dissertation, St. John's University, Queens, NY. Tranel, D., Damasio, A., \& Damasio, H. (1988). Intact recognition of facial expression, gender, and age in patients with impaired recognition of face identity. Neurology, 38, 690-696.

(Manuscript received October 7, 1991.) 